\title{
INEQUALITIES RELATED TO A CERTAIN INEQUALITY USED IN THE THEORY OF DIFFERENTIAL EQUATIONS
}

\author{
B. G. PACHPATTE
}

\begin{abstract}
In this paper we establish some new integral and finite difference inequalities related to a certain integral inequality used in the theory of differential equations. The inequalities obtained here can be used as handy tools in the theory of some new classes of integral and sum-difference equations.
\end{abstract}

\section{Introduction}

Recently, in [11-14] the present author has established a number of new inequalities related to the following inequality.

Theorm A. Let $y$ and $f$ be real-valued nonnegative continuous functions defined for $t \in R_{+}=[0, \infty)$. If

$$
y^{2}(t) \leq c^{2}+2 \int_{0}^{t} f(s) y(s) d s,
$$

for $t \in R_{+}$, where $c \geq 0$ is a constant, then

$$
y(t) \leq c+\int_{0}^{t} f(s) d s
$$

for $t \in R_{+}$.

In the literature there are many papers which make use of this inequality very frequently to study the different properties of the solutions of various nonlinear differential equations, see $[1,3-8,11-15]$ and the references given therein. The importance of this inequality lies in its successful utilization to the situations for which the other available inequalities do not apply directly. The aim of the present paper is to establish some new integral and sum-difference inequalities which claim their origin to the inequality given in Theorem A. The inequalities established here can be used as tools in the study of certain

Received Auguest 23, 1995.

1991 Mathematics Subject Classification. Primary 26D15, Secondary 26D20.

Key words and phrases. Inequalities, theory of differential equations, sum-difference equations, submultiplicative, bounds on the solutions. 
new classes of integral and sum-difference equations. We aslo present, some immediate applications to convey usefulness of our results to the literature.

\section{Statement of Results}

In what follows, we use the following definitions and notations for simplification of details of presentation. Let $R$ denotes the set of real numbers, $R_{+}=[0, \infty)$ and $N_{0}=$ $\{0,1,2, \ldots\}$. For any function $u(n)$ defined for $n \in N_{0}$, we define the operator $\Delta$ by $\Delta u(n)=u(n+1)-u(n)$. For $m>n, m, n \in N_{0}$ and any function $p(n)$ defined for $n \in N_{0}$, we use the usual conventions

$$
\sum_{s=m}^{n} p(s)=0, \prod_{s=m}^{n} p(s)=1 .
$$

For some suitable functions $a, b, y$ and $t \in R_{+}, n \in N_{0}$, we set :

$$
\begin{aligned}
L[t, a, b, y] & =\left(\int_{0}^{t} a(\sigma) y(\sigma) d \sigma\right)\left(\int_{0}^{t} b(\sigma) y(\sigma) d \sigma\right), \\
M[n, a, b, y] & =\left(\sum_{t=0}^{n-1} a(t) y(t)\right)\left(\sum_{t=0}^{n-1} b(t) y(t)\right) .
\end{aligned}
$$

Our main results are given in the following theorem.

Theorem 1. Let $y, a, b, h$ be real-valued nonegative continuous functions defined for $t \in R_{+}$and $c$ be a nonnegative real constant.

$\left(a_{1}\right)$ Let $f: R_{+}^{2} \rightarrow R+$ be a continuous function which satisfies the condition

$$
0 \leq f\left(t, u_{1}\right)-f\left(t, u_{2}\right) \leq k\left(t, u_{2}\right)\left(u_{1}-u_{2}\right),
$$

for $t \in R_{+}$and $u_{1} \geq u_{2} \geq 0$, where $k: R_{+}^{2} \rightarrow R_{+}$is a continuous function. If

$$
y^{2}(t) \leq c^{2}+2 L[t, a, b, y]+2 \int_{0}^{t} y(s) f(s, y(s)) d s,
$$

for $t \in R_{+}$, then

$$
y(t) \leq Q(t)\left[c+\int_{0}^{t} f(s, c Q(s)) \exp \left(\int_{s}^{t} k(\sigma, c Q(\sigma) Q(\sigma) d \sigma) d s\right]\right.
$$

for $t \in R_{+}$, where

$$
Q(t)=\exp \left(\int_{0}^{t}\left[a(s)\left(\int_{0}^{s} b(\sigma) d \sigma\right)+b(s)\left(\int_{0}^{s} a(\sigma) d \sigma\right)\right] d s\right),
$$

for $t \in R_{+}$. 
$\left(a_{2}\right)$ Let $W(y)$ be a real-valued continuous, nondecreasing and submultiplicative function defined on the interval $I=\left[y_{0}, \infty\right)$ and $W(y)>0$ on $\left(y_{0}, \infty\right), y_{0} \geq 0$ is a real constant, $W\left(y_{0}\right)=0$. If

$$
y^{2}(t) \leq c^{2}+2 L[t, a, b, y]+2 \int_{0}^{\ell} h(s) y(s) W(y(s)) d s
$$

for $t \in R_{+}$, then for $0 \leq t \leq t_{1}$

$$
y(t) \leq Q(t) \Omega^{-1}\left[\Omega(c)+\int_{0}^{t} h(s) W(Q(s)) d s\right]
$$

where $Q(t)$ is defined by (2.4) and

$$
\Omega(r)=\int_{r_{0}}^{r} \frac{d s}{W(s)}, r \geq r_{0} \text { with } r_{0}>y_{0},
$$

$\Omega^{-1}$ is the inverse of $\Omega$ and $t_{1} \in R_{+}$be chosen so that

$$
\Omega(c)+\int_{0}^{t} h(s) W(Q(s)) d s \in \operatorname{Dom}\left(\Omega^{-1}\right)
$$

for $0 \leq t \leq t_{1}$.

$\left(a_{3}\right)$ Let $w(t, r)$ be a real-valued nonnegative continuous function defined for $t \in R_{+}$, $0 \leq r<\infty$, and monotone nondecreasing with respect to $r$ for any fixed $t \in R_{+}$. If

$$
y^{2}(t) \leq c^{2}+2 L[t, a, b, y]+2 \int_{0}^{t} y(s) w(s, y((s)) d s,
$$

for $t \in R_{+}$, then

$$
y(t) \leq Q(t) r(t), t \in R_{+},
$$

where $Q(t)$ is defined by (2.4) and $r(t)$ is a maximal solution of

$$
r^{\prime}(t)=w(t, Q(t) r(t)), r(0)=c,
$$

for $t \in R_{+}$.

The discrete analogues of the results given in Therorem 1 are established in the following theorem.

Theorem 2. Let $y, a, b, h$ be real-valued nonnegative functions defined for $n \in N_{0}$ and $c$ be a nonnegative real constant.

$\left(b_{1}\right)$ Let $g: N_{0} \times R_{+} \rightarrow R_{+}$be a function which satisfies the condition

$$
0 \leq g\left(n, u_{1}\right)-g\left(n, u_{2}\right) \leq q_{1}\left(n, u_{2}\right)\left(u_{1}-u_{2}\right)
$$


for $n \in N_{0}$ and $u_{1} \geq u_{2} \geq 0$, where $q(n, r)$ is a real-valued nonegative function defined for $n \in N_{0}, r \in R_{+}$. If

$$
y^{2}(n) \leq c^{2}+2 M[n, a, b, y]+2 \sum_{t=0}^{n-1} y(t) g(t, y(t)),
$$

for $n \in N_{0}$, then

$$
y(n) \leq P(n)\left[c+\sum_{s=0}^{n-1} g(s, c P(s)) \prod_{t=s+1}^{n-1}[1+q(t, c P(t)) P(t)]\right],
$$

for $n \in N_{0}$, where

$$
P(n)=\prod_{s=0}^{n-1}\left[1+a(s)\left(\sum_{t=0}^{s-1} b(t)\right)+b(s)\left(\sum_{t=0}^{s} a(t)\right)\right]
$$

for $n \in N_{0}$.

$\left(b_{2}\right)$ Let $W, \Omega, \Omega^{-1}$ be as in $\left(a_{2}\right)$ in. Theorem 1. If

$$
y^{2}(n) \leq c^{2}+2 M[n, a, b, y]+2 \sum_{t=0}^{n-1} h(t) y(t) W(y(t))
$$

for $n \in N_{0}$, then for $0 \leq n \leq n_{1}$,

$$
y(n) \leq P(n) \Omega^{-1}\left[\Omega(c)+\sum_{s=0}^{n-1} h(s) W(P(s))\right]
$$

where $P(n)$ is defined by (2.14) and $n_{1} \in N_{0}$ be chosen so that

$$
\Omega(c)+\sum_{s=0}^{n-1} h(s) W(P(s)) \in \operatorname{Dom}\left(\Omega^{-1}\right),
$$

for $n \in N_{0}$ and $0 \leq n \leq n_{1}$.

$\left(b_{3}\right)$ Let $w(n, r)$ be a real-valued nonncgative funciton defincd for $n \in N_{0}, r \in R_{+}$, and monotone nondecrcasing ?ith respect to $r$ for any fixed $n \in N_{0}$. If

$$
y^{2}(n) \leq c^{2}+2 M[n, a, b, y]+2 \sum_{t=0}^{n-1} y(t) w(t, y(t))
$$

for $n \in N_{0}$, then

$$
y(n) \leq P(n) r(n), n \in N_{0},
$$

where $P(n)$ is defined by (2.14) and $r(n)$ is a solution of

$$
\Delta r(n)=w(n, P(n) r(n)), r(0)=c
$$


for $n \in N_{0}$.

\section{Proof of Theorem 1}

$\left(a_{1}\right)$ We first assume that $c>0$ and define a function $z(t)$ by the right side of (2.2). Now differentiating $z(t)$ and using the fact that $y(t) \leq \sqrt{z(t)}$ we observe that

$$
\left.z^{\prime}(t) \leq 2 \sqrt{z(t)}\left[a(t) \int_{0}^{t} b(\sigma) \sqrt{z(\sigma)} d \sigma\right)+b(t)\left(\int_{0}^{t} a(\sigma) \sqrt{z(\sigma)} d \sigma\right)+f(t, \sqrt{z(t)})\right] .
$$

Differentiating $\sqrt{z(t)}$, and then using (3.1) we have

$$
\begin{aligned}
\frac{d}{d t}(\sqrt{z(t)}) & =\frac{z^{\prime}(t)}{2(\sqrt{z(t)}} \\
& \leq\left[a(t)\left(\int_{0}^{t} b(\sigma) \sqrt{z(\sigma)} d \sigma\right)+b(t)\left(\int_{0}^{t} a(\sigma) \sqrt{z(\sigma)} d \sigma\right)+f(t, \sqrt{z(t)})\right] .
\end{aligned}
$$

By setting $t=s$ in (3.2) and integrating it from 0 to $t$ we have

$$
\sqrt{z(t)} \leq m(t)+\int_{0}^{t}\left[a(s)\left(\int_{0}^{s} b(\sigma) \sqrt{z(\sigma)} d \sigma\right)+b(s)\left(\int_{0}^{s} a(\sigma) \sqrt{z(\sigma)} d \sigma\right)\right] d s
$$

where

$$
m(t)=c+\int_{0}^{t} f(s, \sqrt{z(s)}) d s .
$$

Since $m(t)$ is positive and monotone nondecreasing for $t \in R_{+}$, from (3.3) we observe that

$$
\frac{\sqrt{z(t)}}{m(t)} \leq 1+\int_{0}^{t}\left[a(s)\left(\int_{0}^{s} b(\sigma) \frac{\sqrt{z(\sigma)}}{m(\sigma)} d \sigma\right)+b(s)\left(\int_{0}^{s} a(\sigma) \frac{\sqrt{z(\sigma)}}{m(\sigma)} d \sigma\right)\right] d s .
$$

Define a function $v(t)$ by the right side of (3.5). Differentiating $v(t)$ and then using (3.5) and the fact that $v(t)$ is monotone nondecreasing for $t \in R_{+}$we observe that

$$
v^{\prime}(t) \leq\left[a(t)\left(\int_{0}^{t} b(\sigma) d \sigma\right)+b(t)\left(\int_{0}^{t} a(\sigma) d \sigma\right)\right] v(t)
$$

The inequality (3.6) implies the estimate

$$
v(t) \leq Q(t), t \in R_{+}
$$

where $Q(t)$ is defined by (2.4). From (3.5) and (3.7) we have

$$
\sqrt{z(t)} \leq Q(t) m(t), t \in R_{+} .
$$


Define a function $u(t)$ by

$$
u(t)=\int_{0}^{t} f(s, \sqrt{z(s)}) d s .
$$

From (3.9), (3.8) and using the fact that $m(t)=c+u(t)$ from (3.4) and the condition (2.1) we observe that

$$
\begin{aligned}
u^{\prime}(t) & \leq f(t, Q(t)(c+u(t))) \\
& =f(t, c Q(t)+Q(t) u(t))-f(t, c Q(t))+f(t, c Q(t)) \\
& \leq k(t, c Q(t)) Q(t) u(t)+f(t, c Q(t)) .
\end{aligned}
$$

The inequality (3.10) implies the estimate

$$
u(t) \leq \int_{0}^{t} f(s, c Q(s)) \exp \left(\int_{s}^{t} k(\sigma, c Q(\sigma)) Q(\sigma) d \sigma\right) d s .
$$

Using (3.11) in $m(t)=c+u(t)$ we have

$$
m(t) \leq c+\int_{0}^{t} f(s, c Q(s)) \exp \left(\int_{s}^{t} k(\sigma, c Q(\sigma)) Q(\sigma) d \sigma\right) d s .
$$

Now the required inequality in (2.3) follows by using (3.12) in (3.8) and then using the fact that $y(t) \leq \sqrt{z(t)}$.

If $c$ is nonnegative, we can carry out the above procedure with $c+\epsilon$ instead of $c$, where $\epsilon>0$ is an arbitrary small constant, and subsequently pass to the limit $\in \rightarrow 0$ to obtain (2.3). This completes the proof of $\left(a_{1}\right)$.

$\left(a_{2}\right)$ Assuming that $c$ is positive and defining a function $z(t)$ by the right side of $(2.5)$ and the function $m(t)$ by

$$
m(t)=c+\int_{0}^{t} h(s) W(\sqrt{z(s)}) d s,
$$

and following the same steps as in the proof of $\left(a_{1}\right)$ we have

$$
\sqrt{z(t)} \leq Q(t) m(t), t \in R_{+},
$$

where $Q(t)$ is defined by (2.4). From (3.13),(3.14) and using the conditions on $W$ we observe that

$$
m^{\prime}(t) \leq h(t) W(Q(t)) W(m(t))
$$

From (2.7) and (3.15) we observe that

$$
\frac{d}{d t} \Omega(m(t)) \leq h(t) W(Q(t))
$$

Integrating both sides of (3.16) from 0 to $t$ we have

$$
\Omega(m(t)) \leq \Omega(c)+\int_{0}^{t} h(s) W(Q(s)) d s .
$$


From (3.17) we have

$$
m(t) \leq \Omega^{-1}\left[\Omega(c)+\int_{0}^{t} h(s) W(Q(s)) d s\right]
$$

Using (3.18) in (3.14) and then the fact that $y(t) \leq \sqrt{z(t)}$ we get the required inequality in (2.6). The subdomain for $t \in R_{+}$is obvious. The proof of the case when $c$ is nonnegative can be completed as mentioned in the proof of $\left(a_{1}\right)$.

$\left(a_{3}\right)$ Assuming that $c$ is positive and defining a function $z(t)$ by the right. side of (2.8) and the function $m(t)$ by

$$
m(t)=c+\int_{0}^{t} w(s, \sqrt{z(s)}) d s,
$$

and following the same steps as in the proof of $\left(a_{1}\right)$ we have

$$
\sqrt{z(t)} \leq Q(t) m(t), t \in R_{+} .
$$

where $Q(t)$ is defined by (2.4). From (3.19) and (3.20) we observe that

$$
m^{\prime}(t) \leq w(t, Q(t) m(t))
$$

Now a suitable application of the basic comparison theorem due to Conti (see $[2$, p.35] or [16] to (3.21) and (2.10) yieds

$$
m(t) \leq r(t), t \in R_{+},
$$

where $r(t)$ is a maximal solution of (2.10). Using (3.22) in (3.20) and then the fact that $y(t) \leq \sqrt{z(t)}$, we get the desired inequality in (2.9). The proof of the case when $c$ is nonnegative can be completed as mentioned in the proof of $\left(a_{1}\right)$.

\section{Proof of Theorem 2}

$\left(b_{1}\right)$ Assume that $c>0$ and define a function $z(n)$ by the right side of (2.12). From the definition of $z(n)$ and making use of the formula

$$
\Delta[u(n) v(n)]=u(n) \Delta v(n)+v(n+1) \Delta u(n),
$$

and the fact that $y(n) \leq \sqrt{z(n)}$ we observe that

$$
\Delta z(n) \leq 2 \sqrt{z(n)}\left[a(n)\left(\sum_{t=0}^{n-1} b(t) \sqrt{z(t)}\right)+b(n)\left(\sum_{t=0}^{n} a(t) \sqrt{z(t)}\right)+g(n, \sqrt{z(n)})\right] .
$$

It is easy to observe that

$$
\Delta(\sqrt{z(n)})=\frac{z(n+1)-z(n)}{\sqrt{z(n+1)}+\sqrt{z(n)}} \leq \frac{\Delta z(n)}{2 \sqrt{z(n)}}
$$


Here in the last step we have used the fact that $\sqrt{z(n)} \leq \sqrt{z(n+1)}$. By using (4.1) in (4.2) we have

$$
\Delta(\sqrt{z(n)}) \leq\left[a(n)\left(\sum_{t=0}^{n-1} b(t) \sqrt{z(t)}\right)+b(n)\left(\sum_{t=0}^{n} a(t) \sqrt{z(t)}\right)+g(n, \sqrt{z(n)})\right] .
$$

By taking $n=s$ in (4.3) and summing both sides of (4.3) from $s=0$ to $n-1$ we have

$$
\sqrt{z(n)} \leq m(n)+\sum_{s=0}^{n-1}\left[a(s)\left(\sum_{t=0}^{s-1} b(t) \sqrt{z(t)}\right)+b(s)\left(\sum_{t=0}^{s} a(t) \sqrt{z(t)}\right)\right]
$$

where

$$
m(n)=c+\sum_{s=0}^{n-1} g(s, \sqrt{z(s)}) .
$$

Since $m(n)$ is positive and monotone nondecreasing for $n \in N_{0}$, from (1.4) we observe that

$$
\frac{\sqrt{z(n)}}{m(n)} \leq 1+\sum_{s=0}^{n-1}\left[a(s)\left(\sum_{t=0}^{s-1} b(t) \frac{\sqrt{z(t)}}{m(t)}\right)+b(s)\left(\sum_{t=0}^{s} a(t) \frac{\sqrt{z(t)}}{m(t)}\right)\right] .
$$

Define a function $v(n)$ by the right side of (4.6). From the definition of $v(n)$ and using (4.6) and the fact that $v(n)$ is monotone nondecreasing for $n \in N_{0}$, wc observe that

$$
\Delta v(n) \leq\left[a(n)\left(\sum_{t=0}^{n-1} b(t)\right)+b(n)\left(\sum_{i=0}^{n} a(t)\right)\right] v(n) .
$$

The incquality (4.7) implies the estimate (see [10])

$$
v(n) \leq P(n), n \in N_{0},
$$

where $P(n)$ is defined by (2.14). From (4.6) and (4.8) we obtain

$$
\sqrt{z(n)} \leq P(n) m(n), n \in N_{0} \text {. }
$$

Define a function $u(n)$ by

$$
u(n)=\sum_{s=0}^{n-1} g(s, \sqrt{z(s)}) .
$$

From (4.10), (4.9) and using the fact that $m(n)=c+u(n)$ from (4.5) and the condition (2.11) we observe that

$$
\begin{aligned}
\Delta u(n) & \leq g(n, P(n)(c+u(n))) \\
& =g(n, c P(n)+P(n) u(n))-g(n, c P(n))+g(n, c P(n)) \\
& \leq q(n, c P(n)) P(n) u(n)+g(n, c P(n)) .
\end{aligned}
$$


The inequality (4.11) implies the estimate (see [10])

$$
u(n) \leq \sum_{s=0}^{n-1} g(s, c P(s)) \prod_{t=s+1}^{n-1}[1+q(t, c P(t)) P(t)]
$$

Using (4.12) in $m(n)=c+u(n)$ we have

$$
m(n) \leq c+\sum_{s=0}^{n-1} g(s, c P(s)) \prod_{t=s+1}^{n-1}[1+q(t, c P(t)) P(t)] .
$$

Now the required inequality in (2.13) follows by using (4.13) in (4.9) and then using the fact that $y(n) \leq \sqrt{-z(n)}$. The proof of the case when $c$ is nonnegative can be completed as mentioned in the proof of $\left(a_{1}\right)$,

The proofs of $\left(b_{2}\right)$ and $\left(b_{3}\right)$ can be completed by following the proof of $\left(b_{1}\right)$ and closely looking at the proofs of $\left(a_{2}\right)$ and $\left(a_{3}\right)$. We note that in the proof of $\left(b_{3}\right)$, we will have to use the basic comparison theorem given by the present author in [9, Theorem 2]. Here we omit the details of $\left(b_{2}\right)$ and $\left(b_{3}\right)$.

\section{Some Applications}

In this section we indicate in brief applications of some of our results to obtain bounds on the solutions" of certain sum-difference and integrodifferential equations. For example, consider the following sum-difference equation

$$
\Delta\left[x^{2}(n)-2\left(\sum_{t=0}^{n-1} F(t, x(t))\right)\left(\sum_{t=0}^{n-1} G(t, x(t))\right)\right]=2 x(n) H(n, x(n)), x(0)=x_{0},
$$

where $F, G, H$ are real-valued functions defined on $N_{0} \times R$. We assume that

$$
\begin{gathered}
|F(n, x(n))| \leq a(n)|x(n)|, \\
|G(n, x(n))| \leq b(n)|x(n)|, \\
|H(n, x(n))| \leq w(n,|x(n)|),
\end{gathered}
$$

where $a, b, w$ are as defined in Theorem 2. It is easy to observe that if $x(n)$ is a solution of (5.1), then it is also a solution of the equivalent sum-difference equation

$$
x^{2}(n)=x_{0}^{2}+2\left(\sum_{t=0}^{n-1} F(t, x(t))\right)\left(\sum_{t=0}^{n-1} G(t, x(t))\right)+2 \sum_{t=0}^{n-1} x(t) H(t, x(t)) .
$$

Using (5.2)-(5.4) in (5.5) we have

$$
|x(n)|^{2} \leq\left|x_{0}\right|^{2}+2\left(\sum_{t=0}^{n-1} a(t)|x(t)|\right)\left(\sum_{t=0}^{n-1} b(t)|x(t)|\right)+2 \sum_{t=0}^{n-1}|x(t)| w(t,|x(t)|) .
$$


Now an application of Theorem $2\left(b_{3}\right)$ yields

$$
|x(n)| \leq P(n) r(n), n \in N_{0},
$$

where $P(n)$ is defined by $(2.14)$ and $r(n)$ is a solution of the difference equation (2.19) with $c=\left|x_{0}\right|$. The inequality (5.7) gives the bound on the solution $x(n)$ of equation (5.1) in terms of the known functions and the solution $r(n)$ of (2.19). If the solution $r(n)$ of (2.19) is bounded and $P(n)$ is finite, then (5.7) implics that the solution $x(n)$ of $(5.1)$ is bounded for $n \in N_{0}$.

We also note that the inequality given in Theorem $1\left(a_{3}\right)$ can be used to obtain bound on the solution of the following integrodifferential equation

$$
\frac{d}{d t}\left[x^{2}(t)-2\left(\int_{0}^{\imath} F(s, x(s)) d s\right)\left(\int_{0}^{t} G(s, x(s)) d s\right)\right]=2 x(t) H(t, x(t)), x(0)=x_{0},
$$

under some suitable conditions on the fuctions involved in (5.8) similar to that of given in (5.2)-(5.4). For inequalities similar to that of given in Theorems 1 and 2 and their applications, see [11-14].

\section{References}

[1] V. Barbu, Differential Equations, Ed. Junimea, Iasi, 1985. [In Romanian].

[2] F. Brauer, "The use of comparison theorems for ordinary differential equations," Stability problems of solutions of differential equations, Proc. of NATO Advanced Study Inslitute, Padua, Italy, 1965, 29-50.

[3] C. M. Dafermos, "The second law of thermodynamics and stability," Arch. Rational Mech. Anal., 70(1979), 167-179.

[4] S. S. Dragomir, "The gronwall type Lemmas and Applications," Monogrfii Matcmatice, Univ. Timisoara, 29, 1987.

[5] A. Haraux, "Nonlinear Evolution Equations:Global Behavior of solutions," Lecture Notes in Mathematics, Springer-Verlag, Berlin, New York 811, 1981.

[6] D. S. Mitrinović and J. L. Pečarić, Differential and Intcgral Incqualitics, Naucna Kujiga Belgrade, 1988.

[7] S. N. Olekhnik, "Boundeness and unboundedness of solutions of some systems of ordinary differential equations," Vestrik Moskov. Univ. Mat., 27(1972), 34-44.

[8] L. Ou-Iang, "The boundedness of solutions of linear differnctial cquations $y^{\prime \prime}+\Lambda(t) y=0$," Shuxue Jinzhan 3(1957), 409-415.

[9] B. G. Pachpatte, "Finite-difference inequalities and an extension of Lyapunov's method," Michigan Math. J., 18(1971), 385-391.

[10] B. G. Pachpatte, "On the discrete generalizations of Gronwall's inequality," J. Indian Math. Soc., 37(1973), 147-156.

[11] B. G. Pachpatte, "On a certain inequality arising in the theory of differential equations," J. Math. Anal. Appl., 182(1994), 143-157.

[12] B. G. Pachpatte, some new finite difference inequalitics, Computcrs Math. Applic., 28 (1994), 227-273. 
[13] B. G. Pachpatte, "On some fundamental integral inequalities arising in the theory of differential equations", Cninese J. Math. 22(1994), 261-273.

[14] B. G. Pachpatte, "On some new inequalities related to certain inequalities in the theory of differential equations", J. Math. Anal. Appl., 189(1995), 128-144.

[15] M. Tsutsumi and I. Fukunda, "On solutions of the derivative nonlinear Schrödinger equation: Existence and uniqueness theorem," Funkcial. Ekvac., 23(1980), 259-277.

[16] W. Walter, Differential and Integral Inequalities, Springer-Verlag, Berlin, New York, 1970. Department of Marathematics, Marathwada University, Aurangabad 431004 , (Maharashtra)
India. 